\title{
Review on carbon nanotubes and carbon nanotube bundles for gas/ion separation and water purification studied by molecular dynamics simulation
}

\author{
S. M. Fatemi ${ }^{1}$ - M. Foroutan ${ }^{1}$
}

Received: 19 April 2015/Revised: 10 September 2015/Accepted: 1 December 2015/Published online: 15 December 2015

(C) Islamic Azad University (IAU) 2015

\begin{abstract}
Water and air pollutants have huge impacts on the entire living system. In addition, the newly emerging nanopollutants, increasing global warming, and consequent climate changes are posing major threats to the freshwater or fresh air availability. This has made it urgent to invent an appropriate water/air treatment technology that removes nanopollutants and also desalinates water to a significant extent. Carbon-based nanomaterials have generated marvelous interest in a wide range of research activities due to their high adsorption capacities. Carbon nanotubes, carbon nanotube bundles, and related materials have potential applications in gas/ion separation and water purification. Recently, gas/ion separation and water purification through carbon-based nanomaterials have received increasing attention. This review summarizes the properties of carbon nanotubes and carbon nanotube bundles related to gas separation, ion separation, and water purification using molecular dynamics simulations. Membranes-based carbon nanomaterials show promise of water purification and gas separation. These attractive properties of carbon nanotubes-based and carbon nanotube bundles-based nanomaterials make them proper candidates for gas separation, gas molecular-sieving processes, and desalination purposes in nanoscale dimensions.
\end{abstract}

Keywords Molecular dynamics simulation - Carbon nanotube $\cdot$ Carbon nanotube bundles - Gas separation . Water purification

M. Foroutan

foroutan@khayam.ut.ac.ir

1 Department of Physical Chemistry, School of Chemistry, College of Science, University of Tehran, Tehran, Iran

\section{Introduction}

Carbon-based nanomaterials have become important due to their unique applications in different fields of purification such as gas/ion separation and water purification (Hitsov et al. 2015; Mohammad et al. 2015). Carbon-based nanomaterials with exclusive physical properties including electrical conductivity (Guo et al. 2012), mechanical strength, and optical properties (Ren and Wang 2010) have great significance in generating advancement for researchers. Carbon nanotubes (CNTs) are nanosized allotropes of carbon which were first discovered by Iijima (1991) at Fundamental Research Laboratories of Japan in 1991. Functional, mechanical, electrical, thermal, and optoelectronic properties of CNTs are dependent on the diameter and length of the tubes, the atomic arrangement, and the morphology of the nanostructure (Bandaru 2007). Soon after the discovery of CNTs, different types of them were synthesized such as single-walled and multiwalled CNTs, as well as CNT bundles. In 2006, Kang and coworkers described a nanotube bundle as aligned tubes in a triangular lattice which can form hexagonal rings (Kang et al. 2006). CNT bundles can improve the electrical, mechanical, and thermal properties (Bandaru 2007; Lau et al. 2005) as well as graphene can do. Similar to CNTs, graphene contains $\mathrm{sp}^{2}$-hybridized carbon sheets which are composed of six-member carbon rings. The layers of graphene have a high surface area with a good thermal and electrical conductivity as well as a high Young's modulus (Novoselov et al. 2012). Hence, it was broadly studied in the fields of gas sorption, separation, and storage due to its unique physical and chemical properties (Chen et al. 2012; Kuila et al. 2012; Wei and Qu 2012; Yavari and Koratkar 2012; Fatemi et al. 2015). To date, scientists tried to describe the mechanism of graphene water purification and 
gas/ion separation; nevertheless, the achievement of a single opinion for this purpose is difficult since the various tests have conflicting results. The molecular dynamics (MD) simulation is widely used for theoretical studies of gas/ion separation and water purification. By the use of MD simulations, prediction of the chemical properties of substances and the microscopic interactions between the components of a system is possible. Simulation is a bridge between theory and experiment, and thus, MD simulations can develop microscopic information, including atomic positions and velocities. In this review, recent developments concerning the CNT and CNT bundles for gas/ion separation and water purification obtained by MD simulation are presented. Simulations were carried out in 2014-2015, in the Department of Physical Chemistry, School of Chemistry, College of Science, University of Tehran, Tehran, Iran.

\section{Separation of gaseous mixtures using carbon- based nanomaterials}

\section{Separation of gaseous mixtures using CNTs}

Techniques used to separate gases, either to provide multiple products or to purify a single product, are named as gas separation techniques and are basically dependent on the membranes. Consequently, the improvement in advanced membrane technologies with a specified pore size is necessary to gain a more efficient and cost-effective separation. Polymeric membranes are microporous films possessing uniform pores and act as semipermeable barriers to separate different mixtures using their specific properties such as selectivity and/or permeability. Sometimes, their pores are polluted with chemicals which diminish their performance. Membranes based on CNTs with a unique structure have overcome this problem (Brady-Estévez et al. 2008; Miller et al. 2001; Peng et al. 2007; Srivastava et al. 2004; Thomas and McGaughey 2008; Whitby et al. 2008). In the following, the historical perspective, and then the properties and applications of CNTs in the different gas separation fields will be introduced.

CNTs are cylindrical nanostructures of rolled-up graphene with the outer and inner diameters in the range of 1-3 and 0.4-2.4 nm, respectively. CNTs have good, remarkable electrical and thermal conductivity and are one of the strongest known fibers (Baughman et al. 2002; Jorio et al. 2007). The combination of these properties with their nanoscale dimensions makes CNTs significant materials in a wide range of applications (Fatemi and Foroutan 2015a,
2016; O'connell 2006). However, open-ended CNTs (i.e., having their end-caps etched off) typically have no sufficiently small pores to be selective for gas-phase molecules. Additionally, the existing methods for the synthesis of CNTs are not able to produce uniform CNTs with a given diameter pore, so the nanotubes are produced with different diameters. For instance, Liu et al. (2015) investigated the capability of three types of nanoporous carbons for adsorption of $\mathrm{CH}_{4}$ from $\mathrm{CO}_{2} / \mathrm{CH}_{4}$ mixtures via Monte Carlo simulation. The nanoporous carbons were CNTs, activated carbon fiber (ACF-15), and silicon carbidederived carbon (SiC-DC) which had distinctly different disordered structures. It was found that, by increasing the pressure, the selectivity of amorphous ACF-15 and SiC-DC decreased, while the $\mathrm{CO}_{2}$ selectivity of a $(10,10) \mathrm{CNT}$ was increased as a function of pressure due to the competition and interaction between the adsorbate and adsorbent. It was also found that increasing the concentration of $\mathrm{CO}_{2}$ in the gas phase can increase the $(10,10) \mathrm{CNT}$ selectivity, but it did not change the selectivity of the amorphous ACF-15 and SiC-DC. Additionally, the adsorbate-adsorbate pair provides an adsorbate sieve which became more selective for the $\mathrm{CO}_{2}$ as its concentration increased in the gas phase. Accordingly, at high pressures, as the concentration of $\mathrm{CO}_{2}$ enhanced from 5 to $50 \%$, the selectivity of the $(10,10)$ CNT was almost doubled. Besides this, the effect of temperature was also studied in this process, and the results showed that increase in temperature leads to reduction in the selectivity in all samples. However, the $(7,7) \mathrm{CNT}$, with a diameter of $0.95 \mathrm{~nm}$, had the highest selectivity for $\mathrm{CO}_{2}$ at $0.1 \mathrm{MPa}$ and therefore the maximum adsorption amount of $\mathrm{CO}_{2}$. In a similar research, Surapathi and team (Surapathi et al. 2013) have functionalized CNTs with carboxylic acid and zwitterion groups in simulation calculations. In this calculation, the effect of modification was evaluated by determining the sorption of $\mathrm{CO}_{2}, \mathrm{CH}_{4}$, and $\mathrm{N}_{2}$ at $35^{\circ} \mathrm{C}$ in increasing pressures up to 10 bar. The zwitterion-functionalized CNTs (zwitterion/CNTs) were $\mathrm{CO}_{2}$ selective. It was also found that the gas adsorption of zwitterion/CNTs was dramatically lower than that of carboxylic acid/CNTs. The presence of water on the zwitterion/CNTs surface reduced the sorption even further. In order to reduce the flux of $\mathrm{CO}_{2}$ into the tubes, the existence of three or more zwitterion molecules per tube entrance was essential, and moisture content of the gas phase was quickly sorbed into the zwitterion/CNTs; both of these cases increased the free-energy barrier to $\mathrm{CO}_{2}$ entering the tube and thus reduced the competitive equilibrium adsorption. In another study, to understand the potential of CNTs for flue gas separation, the permeation of $\mathrm{CO}_{2} / \mathrm{N}_{2}$ through CNT membrane was investigated (Ban and Huang 
2012). The adsorption isotherms were calculated by grand canonical Monte Carlo simulation, and the results confirmed that the pure $\mathrm{CO}_{2}$ could be loaded on the CNTs more than pure $\mathrm{N}_{2}$, while a mixture of $\mathrm{CO}_{2} / \mathrm{N}_{2}$ (in a ratio of 1:9) had approximately identical loading in a feed pressure. Furthermore, gas molecules tend to flow on the internal surface of $(10,10) \mathrm{CNT}$ walls. The calculated rate of $\mathrm{N}_{2}$ diffusion was a few times faster than that of $\mathrm{CO}_{2}$, while the $\mathrm{CO}_{2} / \mathrm{N}_{2}$ mixtures had same diffusion coefficients same at high concentrations because of the correlation effect which led to the slowdown of the mobile species and increasing the movement speed of the other tardy component. The experimental results were in agreement with the calculated $\mathrm{N}_{2}$ permeance data. The computed $\mathrm{CO}_{2}$ flux through CNT membranes was higher than that of $\mathrm{N}_{2}$ for single components, while they became nearly identical under flue gas conditions.

Porous CNTs (PCNTs) are one of the CNT categories which possess tailored pores in their sidewalls and recommended as potential membrane materials for high selective gas separation. Bucior et al. (Bucior et al. 2012) compared the obtained results of empirical potentials with quantum mechanical and classical statistical mechanical calculations. PCNTs are very selective to separate the methane from the mixtures of $\mathrm{H}_{2} / \mathrm{CH}_{4}$ as well as $\mathrm{CO}_{2} / \mathrm{CH}_{4}$. In each of these mixtures, $\mathrm{CH}_{4}$ was effectively prevented from entering the pores due to the size exclusion. The initial configuration for the $\mathrm{H}_{2} / \mathrm{CH}_{4}$ mixture is shown in the left-hand panel of Fig. 1.

The permeance rate of hydrogen molecules onto the PCNT pores was very high which prevented the entering of methane molecules. The ending configuration, after the simulation reached an apparent steady state, is shown in the right-hand panel of Fig. 1. It was concluded that the PCNTs are applicable in the matrix of polymer membranes to simultaneously increase the permeance and the selectivity for targeted gas mixtures.

CNTs were also used for adsorption investigation of pure $\mathrm{H}_{2} \mathrm{~S}$ and $\mathrm{SO}_{2}$, and their selectivity was also investigated in the binary mixtures including $\mathrm{H}_{2} \mathrm{~S}-\mathrm{CH}_{4}, \mathrm{H}_{2} \mathrm{~S}-$ $\mathrm{CO}_{2}, \mathrm{SO}_{2}-\mathrm{N}_{2}$, and $\mathrm{SO}_{2}-\mathrm{CO}_{2}$ (Wang et al. 2011). The (20, 20) $\mathrm{CNT}$, which has a larger pore diameter, showed a significantly larger capacity for $\mathrm{H}_{2} \mathrm{~S}$ and $\mathrm{SO}_{2}$ pure gases at $T=303 \mathrm{~K}$, while the $(6,6) \mathrm{CNT}$ with a small pore diameter exhibited the greatest selectivity for binary mixtures containing trace sulfur gases at $T=303 \mathrm{~K}$ and $P=100 \mathrm{kPa}$. The study of the pore size impact on the selective separation of gas mixtures demonstrated that the best pore size for the separation of $\mathrm{H}_{2} \mathrm{~S}-\mathrm{CH}_{4}, \mathrm{H}_{2} \mathrm{~S}-\mathrm{CO}_{2}$, and $\mathrm{SO}_{2}-\mathrm{N}_{2}$ mixtures is $0.81 \mathrm{~nm}$, while it is $1.09 \mathrm{~nm}$ for the $\mathrm{SO}_{2}-\mathrm{CO}_{2}$ mixture. Furthermore, the concentration of sulfur components has a slight effect on the selectivity of CNTs for these binary mixtures. However, with the increase in temperature, the selectivity decreases obviously. In order to improve the adsorption capacities, the surface of CNTs was modified with the functional groups. The site density had the minor impact on the selectivity of $\mathrm{H}_{2} \mathrm{~S}-\mathrm{CO}_{2}$ and $\mathrm{SO}_{2}-\mathrm{CO}_{2}$ mixtures, while with an increase in the site density, the selectivity of $\mathrm{H}_{2} \mathrm{~S}-\mathrm{CH}_{4}$ mixture can improve doubly.

The other types of CNTs were also investigated, in which Liu et al. (2012) selected windowed CNTs (WCNTs: with length $20 \mathrm{~nm}$ ) (Fig. 2a) to separate $\mathrm{CO}_{2}$ from the $\mathrm{CO}_{2} / \mathrm{CH}_{4}$ mixture. So they loaded an appropriate amount of $\mathrm{CH}_{4} / \mathrm{CO}_{2}$ mixture onto the WCNTs (see Fig. 2b); then, they monitored the number of permeate
Fig. 1 Initial (left) and final (right) snapshots for the $\mathrm{H}_{2} / \mathrm{CH}_{4}$ mixture simulation. The final configuration was obtained after 500 ps. The cyan, dark blue, and white balls on the PCNTs represent carbon, nitrogen, and hydrogen atoms, respectively. Reprinted with permission from Bucior et al. (2012)
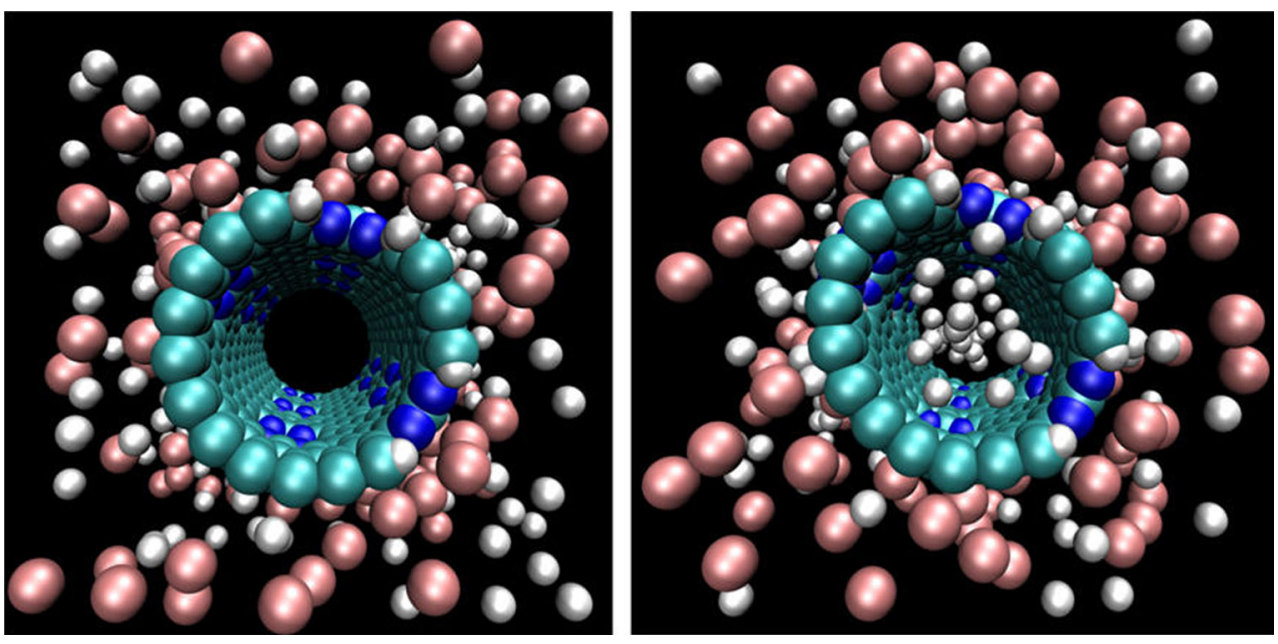
Fig. 2 a 4N4H windows or pores on the wall of the inner tube. b Initial setup of the simulation where the $\mathrm{CO}_{2} / \mathrm{CH}_{4}$ gas mixture is inside the windowed inner tube; on the outside is a pristine tube. The following color code is adopted throughout: carbon (cyan), oxygen (red), hydrogen (white), and nitrogen (blue). Reprinted with permission from Liu et al. (2012)
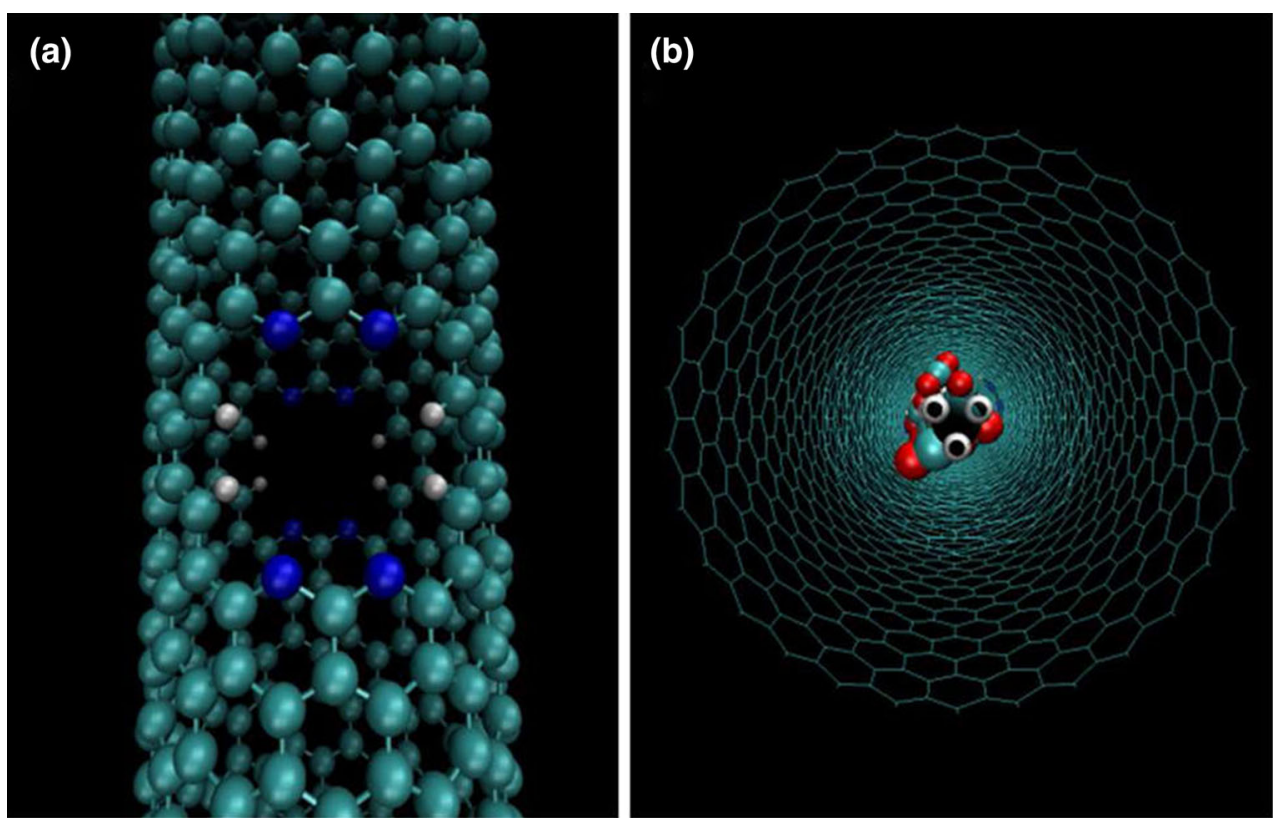

molecules which passed through the nanopores into the space between the two tubes to quantify flux and permeance. Four types of mixtures of $\mathrm{CO}_{2} / \mathrm{CH}_{4}(10,30,50$, and $80 \% \mathrm{CO}_{2}$ ) were studied as a function of pressure which was increased from 80 to 180 bar. In all simulated conditions, the permeated $\mathrm{CO}_{2}$ was only observed; the nitrogen-functionalized windows or pores on the nanotube's wall completely prohibited methane molecules in the accessible time scale while maintaining a fast diffusion rate along the tube.

The estimated time-dependent $\mathrm{CO}_{2}$ permeance ranges from 107 to 105 GPU (Gas Permeation Unit), has been compared with around $100 \mathrm{GPU}$ for typical polymeric membranes. Considering the differences in free-energy barriers of permeation, the $\mathrm{CO}_{2} / \mathrm{CH}_{4}$ selectivity was estimated to be $\sim 108$. Thus, WCNTs can be used as highly efficient membranes, configurable in hollow-fiber-like modules, for $\mathrm{CO}_{2}$ removal from the natural gas.

In another work, Chen et al. (2014) performed a series of MD simulations to investigate the transport properties of hydrogen molecules confined within flexible narrow CNTs. They showed that the hydrogen molecules exhibit three distinct diffusion regimes, namely single-file, Fickian, and ballistic, depending on the value of the Knudsen number. The mean square displacement (MSD) profiles showed that the hydrogen molecules experience three distinct diffusion regimes, namely a short ballistic regime, followed by a subdiffusive regime due to a thermal equilibrium process, and finally an anomalous diffusion regime or a return to a ballistic diffusion regime, depending on the value of Knudsen number. Similar distinct diffusion regions have been observed for the diffusion of colloids and certain lightweight molecules (Jakobtorweihen et al. 2005). In addition, it was shown that with the Knudsen number of less than 1, the tube-wall long-wavelength acoustic phonons-induced Rayleigh traveling wave prompts a longitudinal wave slip and compression-expansion of the hydrogen molecule crowds within the CNT, which leads to a significant increase in the MSD of the molecules. Moreover, MD simulations were used for investigation of the competitive adsorption of gaseous mixture of ethane and ethylene (in a ratio of 1:1) onto the pores of CNTs with different diameters at room temperature (Tian et al. 2013). In this research, the selectivity of narrow CNTs including the $(6,6)$ and $(7,7)$ CNTs, toward ethane molecules, $f_{\text {selec }}$, was 3.1 and 3.7, respectively. The dispersion interaction of the two hydrogen atoms of ethane additionally (compared to ethylene) with the CNTs has a significant role under extreme confinement. Interestingly, the $(8,8) \mathrm{CNT}$ preferred ethylene to ethane with $f_{\text {selec }}=0.6$. For CNTs with a wider dimension, $f_{\text {selec }}$ converged to $\sim 1$. It was suggested that an efficient separation of ethane/ethylene mixture can be done by using membranes of CNT bundles with appropriate diameters. It is worth noting that the $(6,6)$ and $(7,7)$ CNTs favored ethane to ethylene, while the $(8,8)$ CNT preferred ethylene. With further increase of the CNTs diameter, the binding affinities of the two gases to the CNTs become stronger. 
Fig. 3 Snapshots of neon adsorption on the internal and external surfaces of a $(10,10)$ $\mathrm{CNT}$ at $50 \mathrm{~K}$ : a lateral and b front view. Reprinted from Foroutan and Nasrabadi (2010a)

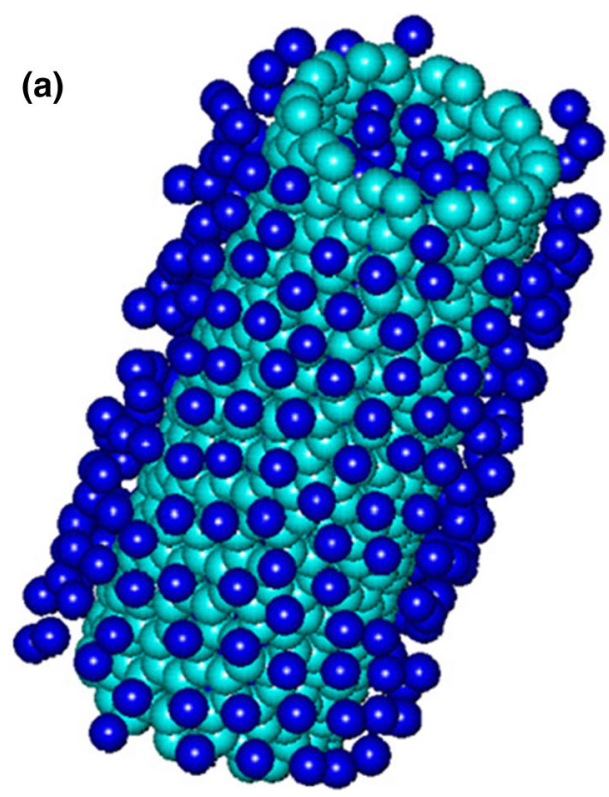

(b)

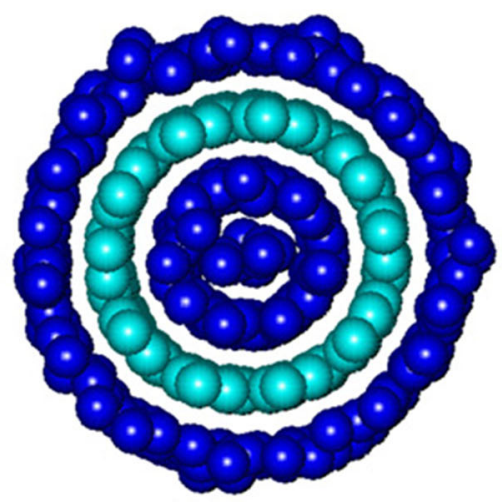

In another publication, neon adsorption on an openended $(10,10)$ CNT was investigated using MD simulations, at $T=50,70$, and $90 \mathrm{~K}$ which are supercritical temperatures of neon (Foroutan and Nasrabadi 2010a). Figure 3 shows snapshots of neon adsorption on the internal (endo) and external (exo) surfaces of nanotube at a temperature of $50 \mathrm{~K}$.

The adsorption was very sensitive to applied temperature in which the increase in temperature reduced the adsorption; the results confirmed this point in a compatible way altogether. The earlier reports (Cheng et al. 2005; Zolfaghari et al. 2007) revealed that CNTs curvature may directly impact the adsorption. Adsorption isotherms, selfdiffusion coefficient, heat of adsorption, and structural properties of neon gas were calculated and evaluated in more detail. A Langmuir shape type I was predicted using all adsorption isotherms at this range of temperature. In evaluating the structural features of the adsorption systems, the best concept of the adsorption process was obtained by the radial distribution function (RDF). This function was defined as the possibility of the presence of neon atoms on the nanotube surface at distance $r$, relative to the expected probability of an entirely random distribution at the same density of neon. As it was found from the heat of adsorption, the adsorption isotherms, and self-diffusion coefficients, once again the RDF plots highlighted this fact that reduction in the temperature leads to more adsorption of neon. This is in agreement with the other experimental and computational published researches (Darkrim et al. 2002; Majidi and Tabrizi 2010), which reported a monotonical increase in the adsorption amount as the temperature

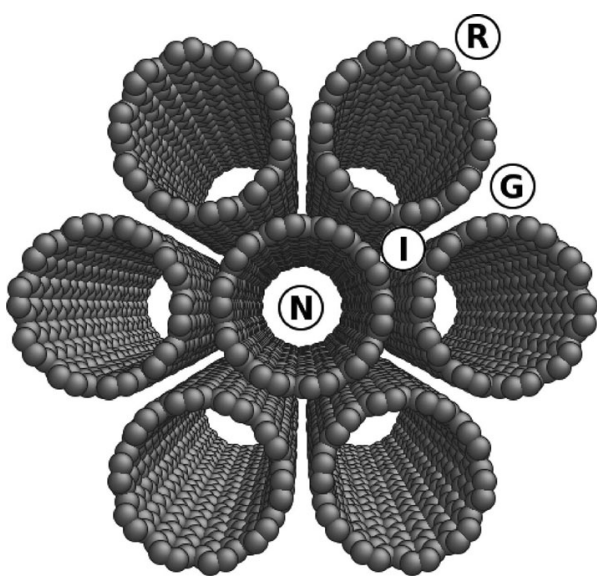

Fig. 4 Four principle adsorption sites of a hexagonal nanotube bundle consists of inner-nanotube sites $(N)$, interstitial sites $(I)$, groove sites $(G)$, and ridge sites $(R)$. Reprinted with permission from Cannon et al. (2012)

decreases. This function advanced a value of unity in the limit of no correlation between investigated particles.

\section{Separation of gaseous mixtures using CNT bundles}

The specific electronic and mechanical properties of CNTs are as a result of their one-dimensional characteristic as well as the particular arrangement of the carbon atoms which constitute their tubular structure (Odom et al. 1998). The arrangement type of carbon atoms may provide chirality and different properties of CNTs including semiconducting, semi-metallic or metallic as well as the 
modified mechanical properties which are independent of the CNTs' chirality. In macroscopic scale, CNT bundles are almost easily grown and formed since CNTs attract each other and cluster into bundles. Bundling can modify the mechanical and electronic properties since the one-dimensionality character of CNTs in form of bundles is reduced and the neighboring tubes interact via van der Waals interaction (Fatemi and Foroutan 2013; Reich et al. 2002; Yu et al. 2000). Therefore, the bundles need to be cracked, and CNTs have to be isolated. Though, the bundling and debundling process is not well understood.

Adsorption onto CNT bundles may find use in various applications such as gas preconcentration and separation, and as a result, it is of great interest to study the adsorption properties of such bundles (Jalili and Majidi 2007a, b; Majidi 2014; Yoo et al. 2002a, b). Four possible adsorption sites can be considered on CNT bundles (see Fig. 4): the hollow interior of nanotubes $(\mathrm{N})$, the interstitial channels between the nanotubes (L), the grooves present on the periphery of a nanotube bundle $(\mathrm{G})$, and the exterior surface of the outermost nanotubes $(\mathrm{R})$.

For example, theoretical calculations showed that, when $\mathrm{He}, \mathrm{H}_{2}$, and $\mathrm{Ne}$ are adsorbed onto the channels of CNTs, all other molecules are too large to enter in these small spaces (Stan et al. 2000a). The interstitial channels are accessible

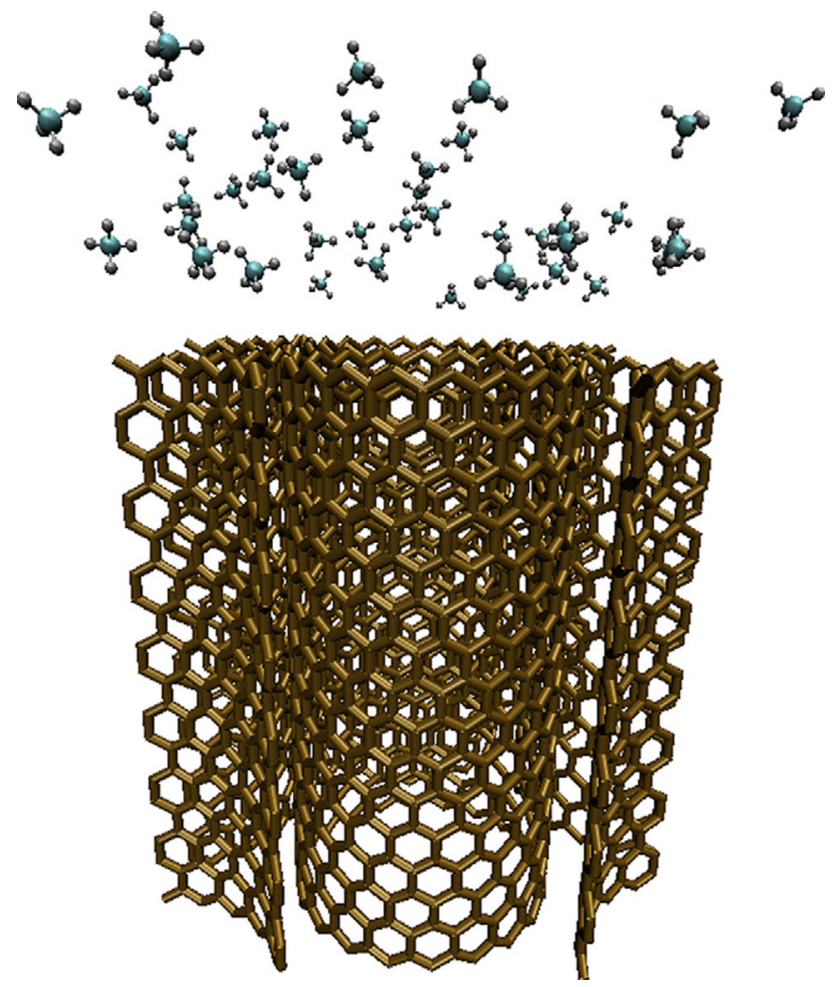

Fig. 5 Snapshot (side view) of a possible initial configuration of the MD simulation. Reprinted from Vela and Huarte-Larrañaga (2011) and the most attractive sites among all charge neutral surfaces to adsorb ${ }^{4} \mathrm{He}, \mathrm{H}_{2}$, and $\mathrm{Ne}$ (Cole et al. 2000; Kahng et al. 2011; Stan et al. 2000b; Weber et al. 2000).

The physisorption of methane over perfect CNT bundles was studied by Larranaga and coworkers (Vela and HuarteLarrañaga 2011). For this aim, the initial run of MD simulation was performed using a total number of 30 methane molecules which were loaded on the upper side of the CNT bundles, at a distance between 3.0 and $10.0 \AA$ (along the Z-axis) (see Fig. 5).

The impact of the CNT diameter on the adsorption capacity of the material was examined, as well as the distribution of the adsorbate by considering CNT bundles with different morphologies. Enhancement of the load capacity was observed as the nanotube diameter was changed, together with a distribution change of the adsorbed molecules. It was found that the porosity has a significant effect on the increase in the storage capacity when CNT bundles are made from the nanotubes with small diameter.

MD simulations were also performed for hydrogen physisorption narrow CNT bundles to explore the possibility of using these materials as potential hydrogen storage materials (Knippenberg et al. 2008). The average heat of adsorption was rapidly decreased with the distance, and increasing $\mathrm{H}_{2}$ loading results in weakening adsorption strength. The average heat of adsorption enhanced slightly with the nanotube thickness; nevertheless, the increase was marginal for effective hydrogen storage. In addition, for a similar average CNT diameter, $\mathrm{H}_{2}$ adsorption of heterogeneous bundles was slightly more than that of homogeneous bundles since the heterogeneous bundles have higher surface area to interact with the $\mathrm{H}_{2}$ molecules. Furthermore, when $\mathrm{H}_{2}$ loading is low, $\mathrm{H}_{2}$ molecules have a tendency to become closer to the CNT bundle walls; on the other hand, if it is high (high loading), a large number of $\mathrm{H}_{2}$ molecules were prevented from the CNT bundles due to the $\mathrm{H}_{2}-\mathrm{H}_{2}$ repulsion as well as the limited accessible sites of adsorption. The $\mathrm{H}_{2}$ molecules at the endohedral sites were unable to penetrate the nanotube walls, and thus, they were completely confined onto the pores of CNTs. Nevertheless, these molecules were not statically adsorbed near the walls. Instead, they move in all the spaces of the endohedral sites to form layered adsorption structures.

In a similar work, the grand canonical Monte Carlo simulations were conducted for light linear alkanes adsorbing onto the closed nanotube bundles to explore these impacts in a systematic manner (Cannon et al. 2012). The results confirmed that the adsorption of alkanes into the grooves of the bundle is preferred along the nanotube axis. Besides this, the grooves of the bundle play a 
significant role in the enhancement of selectivity at lower pressures although at higher pressures those are less important since confinement is practiced at all sites. Albesa and team accomplished Monte Carlo simulations to study the adsorption of single component of pure ethane and ethylene and also equimolar mixtures of them on the bundles of closed single-walled CNTs (Albesa et al. 2012). Two types of nanotube bundles were applied in their simulations, including homogeneous (CNTs with the same diameters) and heterogeneous (CNTs with different diameters). It was illustrated that at the same pressure and temperature (over the entire range), more pure ethane molecules can adsorb on the bundles than pure ethylene molecules. The simulation results for the equimolar mixtures presented that the pressure at which the extreme separation was managed is a very sensitive function to the diameter of the nanotubes present in the bundles. Simulations yield better agreement with single component experimental data using heterogeneous bundles for isotherms and isosteric heats than those obtained using homogeneous bundles. In another study, the storage of $\mathrm{SF}_{6}$ was studied on the bundles of CNTs (Furmaniak et al. 2012). The nanotube bundles were effective $\mathrm{SF}_{6}$ storage vessels, and chirality had no effect on the adsorption. The smallest tubes were the most promising adsorbents for $\mathrm{SF}_{6}$ adsorption accomplished from the mixtures containing low concentration of this gas. In addition, the bundles composed of tubes $(2.7-2.8 \mathrm{~nm}$ diameter), and accessible intestinal porosity was efficient.

Adsorption and separation of binary mixtures of noble gases including Argon (Ar), Krypton (Kr), and Xenon (Xe) on the bundles of $(10,10)$ CNT were simulated by Foroutan and Nasrabadi (2011a). Adsorption energies, diffusion coefficients, activation energies, and RDFs were computed to obtain the thermodynamics, transport, and structural properties of adsorption process. The simulation results of exposing $\mathrm{Ar}-\mathrm{Kr}, \mathrm{Ar}-\mathrm{Xe}$, and $\mathrm{Kr}-\mathrm{Xe}$ mixtures on $(10,10) \mathrm{CNT}$ bundles were calculated at temperatures of 75,150 , and $300 \mathrm{~K}$ and showed that the adsorption amount is strongly affected by the applied temperature. On the other hand, RDF plots obviously showed that when the heavier noble gas is adsorbed more than the lighter one in a selective manner by bundle, the separation of binary gaseous mixture is performable. It was seen that the increase in the applied temperature results in efficient separation. Figure 6 shows the snapshots of adsorption of $\mathrm{Ar}-\mathrm{Kr}, \mathrm{Ar}-$ $\mathrm{Xe}$, and $\mathrm{Kr}-\mathrm{Xe}$ mixtures on a $(10,10) \mathrm{CNT}$ bundle at a temperature of $300 \mathrm{~K}$, in which the green, white, and pink balls represent the $\mathrm{Ar}, \mathrm{Kr}$, and $\mathrm{Xe}$ atoms, respectively.

In order to simultaneously investigate the gas storage and gas filtering capability of CNT bundles, Foroutan and coworkers analyzed the adsorption behavior of a ternary mixture of noble gases on the $(10,10)$ CNT bundles (Foroutan and Nasrabadi 2010b). Using MD simulations, selfdiffusion coefficients, activation and adsorption energies, and RDFs were calculated at different temperatures of 75 , $100,150,200,250$, and $300 \mathrm{~K}$ to evaluate the thermodynamics, transport, and structural characteristics of the adsorption systems. The results illustrated that the CNT bundles adsorbed a greater amount of heavier noble gases in comparison with the lighter ones. This interesting behavior of CNT bundles converts them as proper candidates for gas storage or separation and gas molecular-sieving processes. Figure 7 shows the last snapshot of $\mathrm{Ar}-\mathrm{Kr}-\mathrm{Xe}$ adsorption on a $(10,10)$ CNT bundle at a temperature of $75 \mathrm{~K}$. Two main adsorption sites can be found: first, gas atoms are adsorbed inside the nanotubes (interior space of tubes) distributed rather uniformly, as a coaxial cylinder; and a second (a)

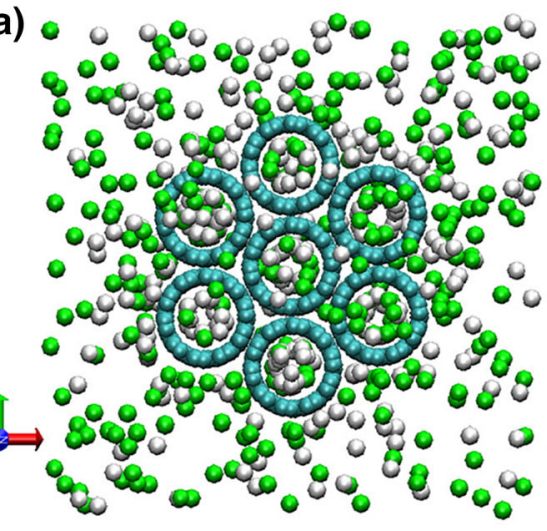

(b)

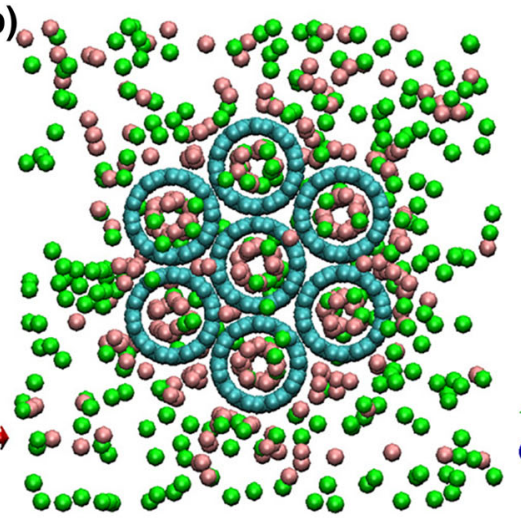

(c)

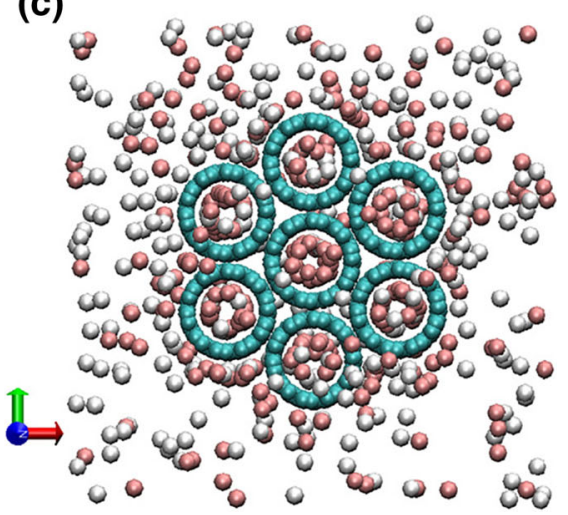

Fig. 6 Snapshots of adsorption of a $\mathrm{Ar}-\mathrm{Kr}$, b Ar-Xe, and $\mathbf{c} \mathrm{Kr}-\mathrm{Xe}$ on a $(10,10) \mathrm{CNT}$ bundle at $T=300 \mathrm{~K}$. Reprinted from Foroutan and Nasrabadi (2011a) 


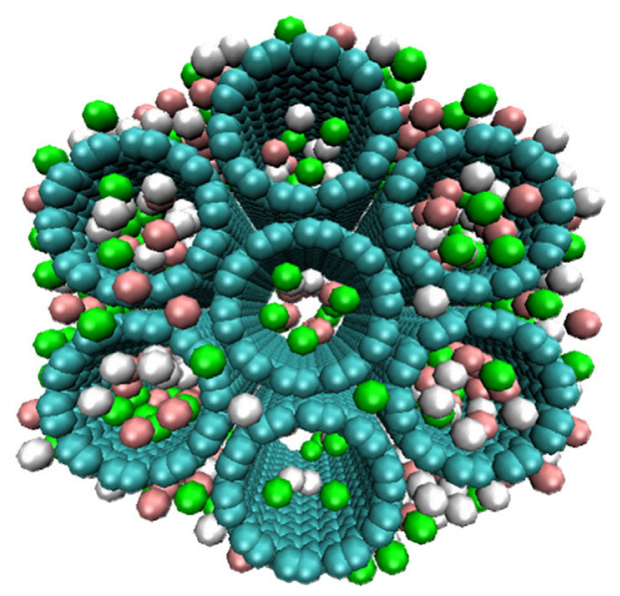

Fig. 7 Adsorption sites of $\mathrm{Ar}-\mathrm{Kr}-\mathrm{Xe}$ mixture inside $(10,10) \mathrm{CNT}$ bundle at $T=75 \mathrm{~K}$. Reprinted from Foroutan and Nasrabadi (2010b)

adsorption site is observed on the external surface of nanotube bundle, while no adsorption was observed in the interstitial threefold pores between the tubes forming the bundle. As shown in Fig. 7, one may think that some gas atoms have been adsorbed in the interstitial threefold pores, but in fact, these atoms have been adsorbed on the upper side of CNTs (external adsorption).

Also Foroutan et al. (Foroutan and Nasrabadi 2012) performed MD simulations to investigate the adsorption of a nitrogen-oxygen mixture (representing air) on $(8,8),(10$, $10)$, and $(12,12)$ CNT bundles as a function of temperature, air loading, and diameter of nanotubes at subcritical $(T=100 \mathrm{~K})$ and supercritical $(T=200$ and $300 \mathrm{~K})$ temperatures of the air. They calculated the RDFs for each carbon of the CNT versus air molecules. The RDF plots emphasized that when the temperature is decreased, the more content of the air is adsorbed, as the height of the peaks are reduced versus temperature increase. This is in agreement with the other computational (Cao and $\mathrm{Wu}$ 2004; Cervellera et al. 2008) works which report monotonical increase in adsorption amount while decreasing the temperature. The same behaviors were also observed for $(8,8)$ and $(12,12)$ bundles, but due to brevity and conciseness they were not given. Also, heat of adsorption, diffusion coefficients, and activation energy were computed for further analysis of the adsorption process. The simulation of exposing air on nanotube bundles showed that the amount of adsorption, heat of adsorption, and diffusion coefficients are strongly influenced by the applied temperature, i.e., along with the temperature increase, the amount of adsorption would be reduced. Furthermore, the results showed that oxygen is selectively adsorbed relative to nitrogen, so CNTs can be considered as promising gas filtering systems in addition to their previous gas storage capability.

\section{Ion separation and water purification using carbon-based nanomaterials}

Growing worldwide population, changes in weather pattern, and demand for improved standards of living all contribute to the shortage of both freshwater and energy. Securing abundant freshwater and energy in economical yet environmentally friendly manners is indeed one of the critical challenges to the society (Shannon et al. 2008; Tour et al. 2010). A combination of water desalination technologies and renewable energy sources will most likely be needed to win such a challenge. Desalination is one of the most promising approaches to freshwater recycle (Amokrane et al. 2015; Cohen-Tanugi et al. 2014; Ghaffour et al. 2013; Porada et al. 2013; Wang and Karnik 2012). As nanotubes are innately insoluble in water (Fatemi and Foroutan 2015b), aqueous dispersion requires modification of the nanotube surface. Dispersion via physical approaches has the advantage of preserving the nanotube conjugated system and hence their electrical properties, and nanotubes have been successfully dispersed in water with the aid of a vast number of different dispersive agents (Fatemi and Foroutan 2014, 2015c). The main aspect of CNT applications is water transport due to understanding of the flow phenomenon (Qin et al. 2011; Whitby and Quirke 2007; Yoo et al. 2015). Since MD simulations predicted the fast water transport through CNTs (Hummer et al. 2001), it attracted attentions (Corry 2008; Yu et al. 2010), and later it was observed in experimental results (Holt et al. 2006; Majumder et al. 2005). Recently, in the published research of Majumder et al. (2008) it was shown that the rate water flows through multiwalled CNTs was four to five orders faster than the predicted rate by macroscopic hydrodynamics. The reason of flow enhancements was the more ordered and stronger hydrogen bonds between the water molecules onto the pores of CNTs than in bulk water that causes concerted and rapid motion along the tube axis. Moreover, the weak interaction between water molecules and hydrophobic surface of CNTs resulted in a smooth nanotube surface with the least coefficient friction (Falk et al. 2010). The synthesis of individual or membrane-incorporated CNTs with an exact diameter and chirality is the challenge since they cannot control the CNTs growth (Hinds et al. 2004). Furthermore, the modification of CNTs is largely restricted to the tube 
Fig. 8 Flow of water inside armchair (a) and zigzag

(b) CNT, outside armchair CNT

(c), and between graphene sheets (d). Reprinted with permission from Falk et al. (2010)

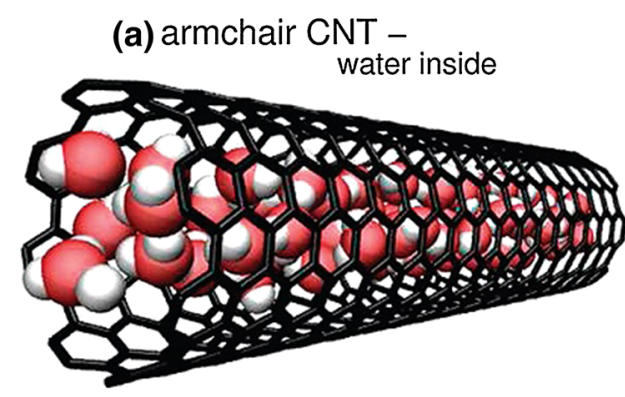

(c) armchair CNT -

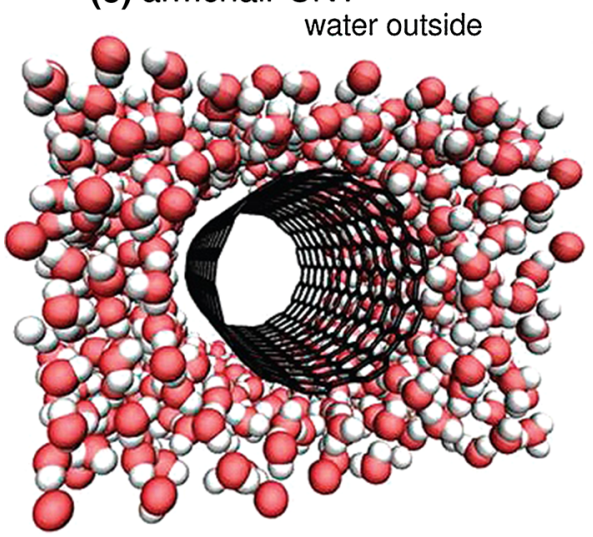

(b) zigzag CNT

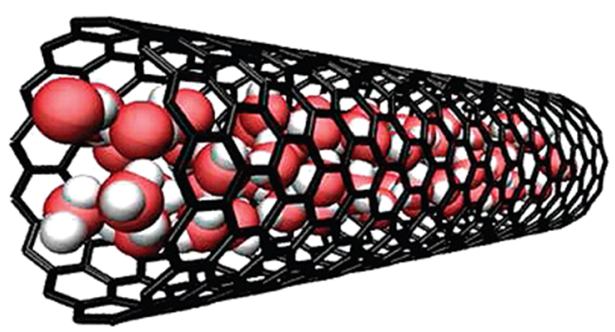

(d) graphene planes

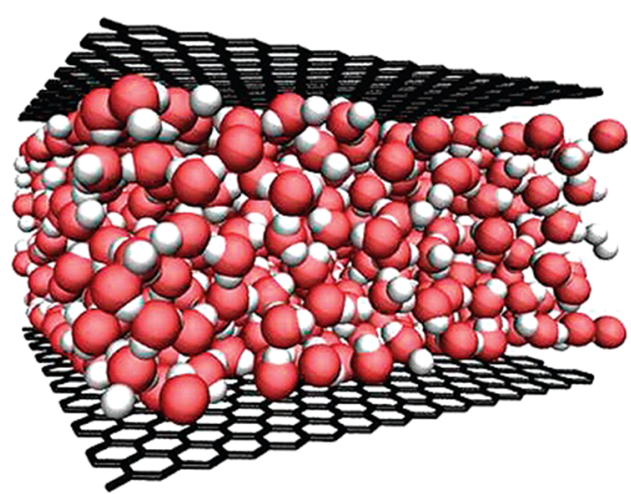

end CNTs due to the $\mathrm{sp}^{2}$ hybridization of carbon along the inert tube surface although novel ways have been projected to overcome this limitation.

Joseph and coworkers displayed that symmetrical modified nanotubes with ionic $-\mathrm{COO}^{-}$and $\mathrm{NH}_{3}{ }^{+}$, which have been attached at the end and onto the pores of tubes, can conduct ions in the presence of an external electric field and achieve the ionic selectivity (Joseph et al. 2003). This result was significant for the specific functionalization of CNTs with small diameter since the presence of charged functional groups on the CNT tip may enable them to control the ionic flow and the exclusion of very small ions. Goldsmith and Martens have also provided some suggestion to improve the ion rejection, in which they ascribed this original mechanism to both direct electrostatic interactions between the charges of CNTs surface and the solution ions and the incidental impact of the water structuring induced by various charge patterns (Goldsmith and Martens 2009). The interfacial friction of water was also studied on graphitic interfaces with different topologies, including the water molecules between the graphene sheets, inside and outside the pores of CNTs (see Fig. 8), with the aim to disentangle confinement and curvature impacts on coefficient friction (Falk et al. 2010).
Based on the obtained results it was found that the friction coefficient exhibits a strong curvature dependence; while friction is independent of confinement for the graphene slab, it decreases with carbon nanotube radius for water inside, but increases for water outside. As a model, the coefficient friction was found to disappear below a threshold diameter for the armchair structure of CNTs. In this way, a structural origin of this curvature dependence, mainly associated with a curvature-induced incommensurability between the water and carbon structures has been reported. These results confirm the recent experiments which reported fast transport of water molecules through the nanometric CNT membranes. In another work, Yzeiri et al. (2014) used classical MD simulations to study nanofluidic properties of porous CNTs. They showed that saturated water vapor which condenses on the porous nanotubes can be absorbed by them and transported in their interior. When these nanotubes are charged and placed in ionic solutions, they can selectively absorb ions in their interior and transport them.

Hughes and team tested the influence of realistic terminations on the water diffusion through the CNTs by computational simulation as well as the interface character in saline medium like seawater concentrations (Hughes 
(a)

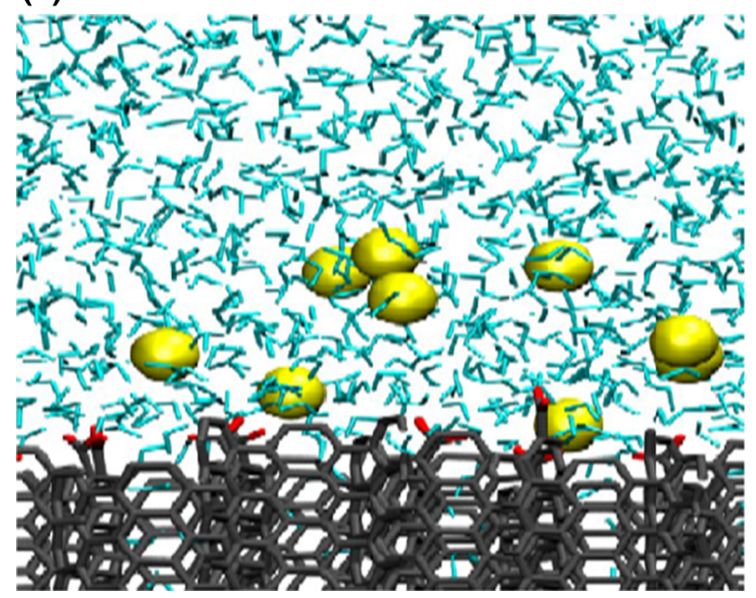

Fig. 9 Snapshots taken from the simulations of a the $(6,6)$ nanotubes with a carboxylate termination with sodium counter-ions and $\mathbf{b}$ the $(6$, 6) nanotubes terminated by hydrogen alone in contact with saline solution. The atoms of carbon, oxygen, hydrogen, sodium cations, (b)

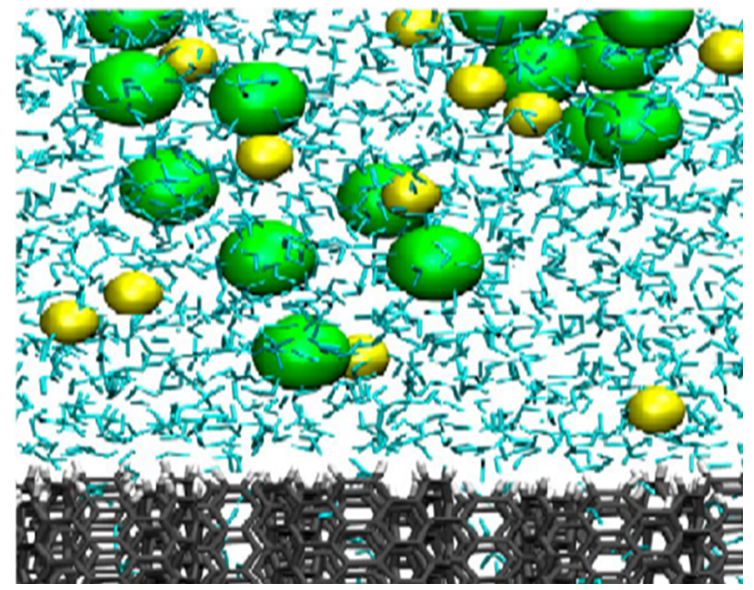

chloride anions, and water molecules are colored gray, red, white, yellow, green, and cyan, respectively. Reprinted with permission from Hughes et al. (2012)
Fig. 10 Last MD snapshot of case $4\left(\sigma=3.36 \mathrm{C} / \mathrm{m}^{2}\right)$ after 2 ns. Reprinted from Foroutan and Nasrabadi (2011b) et al. 2012). Termination of the cleaved CNTs was explored by hydrogen with different functional groups which include hydroxyl, carboxylic acid, and carboxylate anions with sodium counter-cations. Realistic structures reduced the alignment of CNTs within the membrane layer and consequently caused a broader interfacial region. The water diffusion within the finite CNTs was slower than the one observed in the infinite limit, and as the polarity of the end functional groups increased it was generally further reduced. The largest impact occurred for carboxylate 
termination in contact with saline medium, where the ionic solution of sodium ions retarded water diffusion across the interface. In this case, where the CNTs were terminated by hydrogen and a single carboxylate group, the diffusion coefficient was meaningfully reduced. This was due to the presence of the sodium ions around the ends of the nanotubes, meaning that water molecules exiting the nanotubes often become coordinated to the sodium ions, as can be seen from the snapshot in Fig. 9a. In the presence of salt ions, the structure of water around the end of a hydrogenated nanotube seems to increase slightly. The sodium and chloride ions were distributed uniformly throughout the solution, but do not enter the nanotubes. Indeed, they tend to avoid the interfacial region, as illustrated by the configuration shown in Fig. 9b.

Recently, Foroutan confirmed that the charged CNTs can be used as nanoelectrodes to separate the $\mathrm{Na}^{+}$and $\mathrm{Cl}^{-}$ ions from a $\mathrm{NaCl}$ solution as well as for further desalination in a usual nanoscale lab-on-a-chip system (Foroutan and Nasrabadi 2011b). Three different surface charge densities $\left(\sigma=0.84,1.68\right.$, and $\left.3.36 \mathrm{C} / \mathrm{m}^{2}\right)$ were applied on CNTs, and under an external electrical field, positive ions $\left(\mathrm{Na}^{+}\right)$were attracted to negatively charged CNTs and vice versa (see Fig. 10). This external field caused breaking of the ion pairs and consequent desalination, although the quantity of separation depended on the magnitude of the applied charges. Remarkably, by applying the electrical charges on the CNTs, a hydrophilic nature was induced in them, which was completely contrary to their previous hydrophobic nature. Moreover, dependent on the type of applied charge, positive or negative, water molecules orient inversely comparative to the CNT electrodes. Further studies on $\mathrm{Na}^{+}$ions revealed a basic disruption in their hydration shell which occurred in the presence of an external field.

\section{Conclusion}

This review provided an overview of the application of CNTs and CNT bundles in the fields of gas separation and water purification process. Many studies in the field of gas separation/water purification applications of CNTs have been conducted in recent years. In spite of high cost, the use of CNTs as adsorbents will become valuable in the future due to the high adsorption capacities of CNTs compared to the common adsorbents which may offset their high cost. Functionalization of CNT surface can improve their dispersion property in the medium and consequently can enhance the interaction of CNTs with pollutants can increase the removal capacity of CNTs in the preconcentration of pollutants. CNTs have different unique properties which can include the separation ability and nanosize. Simulation calculation results proved that the adsorption and separation of gases can be strongly influenced by the applied temperature. For instance, with an increase in the temperature, the quantity of adsorption decreases while the separation increases. Thus, CNTs provide different applications as adsorbents for gas storage and filtering. The obtained results also illustrated that a charged CNT can be used in separation and encapsulation of ions from a surrounding medium and finally promote the desalination process. Moreover, changes in the charge density of CNT surface modify quantitatively the ion separation. They can thus be applicable as water-purifying membranes (or on the other hand as ion concentrators for the external solution to CNTs) in nanoscale 'lab-on-a-chip' analysis systems.

Acknowledgments The authors wish to extend their sincere gratitude to all who assisted in promoting the present work.

\section{References}

Albesa AG, Rafti M, Rawat DS, Vicente JL, Migone AD (2012) Ethane/ethylene adsorption on carbon nanotubes: temperature and size effects on separation capacity. Langmuir 28:1824-1832

Amokrane M, Sadaoui D, Koutsou C, Karabelas A, Dudeck M (2015) A study of flow field and concentration polarization evolution in membrane channels with two-dimensional spacers during water desalination. J Membr Sci 477:139-150

Ban S, Huang C (2012) Molecular simulation of $\mathrm{CO}_{2} / \mathrm{N}_{2}$ separation using vertically-aligned carbon nanotube membranes. J Membr Sci 417-418:113-118

Bandaru PR (2007) Electrical properties and applications of carbon nanotube structures. J Nanosci Nanotechnol 7:1239-1267

Baughman RH, Zakhidov AA, de Heer WA (2002) Carbon nanotubes-the route toward applications. Science 297:787-792

Brady-Estévez AS, Kang S, Elimelech M (2008) A single-walledcarbon-nanotube filter for removal of viral and bacterial pathogens. Small 4:481-484

Bucior BJ, Chen D-L, Liu J, Johnson JK (2012) Porous carbon nanotube membranes for separation of $\mathrm{H}_{2} / \mathrm{CH}_{4}$ and $\mathrm{CO}_{2} /$ $\mathrm{CH}_{4}$ mixtures. J Phys Chem C 116:25904-25910

Cannon JJ, Vlugt TJ, Dubbeldam D, Maruyama S, Shiomi J (2012) Simulation study on the adsorption properties of linear alkanes on closed nanotube bundles. J Phys Chem B 116:9812-9819

Cao D, Wu J (2004) Self-diffusion of methane in single-walled carbon nanotubes at sub- and supercritical conditions. Langmuir 20:3759-3765

Cervellera VR, Albertí M, Huarte-larrañaga F (2008) A molecular dynamics simulation of air adsorption in single-walled carbon nanotube bundles. Int J Quantum Chem 108:1714-1720

Chen Y, Zhang B, Liu G, Zhuang X, Kang E-T (2012) Graphene and its derivatives: switching $\mathrm{ON}$ and OFF. Chem Soc Rev 41:4688-4707 
Chen B-H, Kung C, Chu I (2014) Mechanisms of hydrogen transport in flexible-wall narrow carbon nanotubes. J Nanomater. doi:10. 1155/2015/959402

Cheng H, Cooper AC, Pez GP, Kostov MK, Piotrowski P, Stuart SJ (2005) Molecular dynamics simulations on the effects of diameter and chirality on hydrogen adsorption in single walled carbon nanotubes. J Phys Chem B 109:3780-3786

Cohen-Tanugi D, McGovern RK, Dave SH, Lienhard JH, Grossman JC (2014) Quantifying the potential of ultra-permeable membranes for water desalination. Energy Environ Sci 7:1134-1141

Cole M, Crespi VH, Stan G, Ebner C, Hartman JM, Moroni S, Boninsegni M (2000) Condensation of helium in nanotube bundles. Phys Rev Lett 84:3883

Corry B (2008) Designing carbon nanotube membranes for efficient water desalination. J Phys Chem B 112:1427-1434

Darkrim FL, Malbrunot P, Tartaglia GP (2002) Review of hydrogen storage by adsorption in carbon nanotubes. Int $\mathrm{J}$ Hydrogen Energy 27:193-202

Falk K, Sedlmeier F, Joly L, Netz RR, Bocquet L (2010) Molecular origin of fast water transport in carbon nanotube membranes: superlubricity versus curvature dependent friction. Nano Lett 10:4067-4073

Fatemi SM, Foroutan M (2013) Structure and dynamics of a nonionic surfactant within a carbon nanotube bundle by molecular dynamics simulation. J Colloid Sci Biotechnol 2:40-44

Fatemi SM, Foroutan M (2014) Study of dispersion of boron nitride nanotubes by triton $\mathrm{X}-100$ surfactant using molecular dynamics simulations. J Theor Comput Chem 13:1450063-1450078

Fatemi SM, Foroutan M (2015a) Recent findings about ionic liquids mixtures obtained by molecular dynamics simulation. J Nanostruct Chem 5:243-253

Fatemi SM, Foroutan M (2015b) Recent developments concerning the dispersion of carbon nanotubes in surfactant/polymer systems by MD simulation. J Nanostruct Chem. Accepted 28 Aug 2015

Fatemi SM, Foroutan M (2015c) Study of dispersion of carbon nanotubes by triton $\mathrm{X}-100$ surfactant using molecular dynamics simulation. J Iran Chem Soc. doi:10.1007/s13738-015-0665-1

Fatemi SM, Foroutan M (2016) Molecular dynamics simulations studies of triton surfactant-wrapped single-walled carbon nanotubes surface. J Adv Phys. doi:10.1166/jap.2016.1251

Fatemi SM, Arabieh M, Sepehrian H (2015) Nanoporous graphene oxide membrane and its application in molecular sieving. Carbon Lett 16:183-191

Foroutan M, Nasrabadi AT (2010a) Molecular dynamics simulation study of neon adsorption on single-walled carbon nanotubes. Phys E 43:261-265

Foroutan M, Nasrabadi AT (2010b) Adsorption behavior of ternary mixtures of noble gases inside single-walled carbon nanotube bundles. Chem Phys Lett 497:213-217

Foroutan M, Nasrabadi AT (2011a) Adsorption and separation of binary mixtures of noble gases on single-walled carbon nanotube bundles. Phys E 43:851-856

Foroutan M, Nasrabadi AT (2011b) Ion-separation and waterpurification using single-walled carbon nanotube electrodes. Desalination 277:236-243

Foroutan M, Nasrabadi AT (2012) Air adsorption and separation on carbon nanotube bundles from molecular dynamics simulations. Comput Mater Sci 61:134-139

Furmaniak S, Terzyk AP, Gauden PA, Kowalczyk P (2012) Simulation of $\mathrm{SF}_{6}$ adsorption on the bundles of single walled carbon nanotubes. Microporous Mesoporous Mater 154:51-55
Ghaffour N, Missimer TM, Amy GL (2013) Technical review and evaluation of the economics of water desalination: current and future challenges for better water supply sustainability. Desalination 309:197-207

Goldsmith J, Martens CC (2009) Molecular dynamics simulation of salt rejection in model surface-modified nanopores. J Phys Chem Lett 1:528-535

Guo W, Liu C, Sun X, Yang Z, Kia HG, Peng H (2012) Aligned carbon nanotube/polymer composite fibers with improved mechanical strength and electrical conductivity. J Mater Chem 22:903-908

Hinds BJ, Chopra N, Rantell T, Andrews R, Gavalas V, Bachas LG (2004) Aligned multiwalled carbon nanotube membranes. Science 303:62-65

Hitsov I, Maere T, De Sitter K, Dotremont C, Nopens I (2015) Modelling approaches in membrane distillation: a critical review. Sep Purif Technol 142:48-64

Holt JK et al (2006) Fast mass transport through sub-2-nanometer carbon nanotubes. Science 312:1034-1037

Hughes ZE, Shearer CJ, Shapter J, Gale JD (2012) Simulation of water transport through functionalized single-walled carbon nanotubes (SWCNTs). J Phys Chem C 116:24943-24953

Hummer G, Rasaiah JC, Noworyta JP (2001) Water conduction through the hydrophobic channel of a carbon nanotube. Nature 414:188-190

Iijima S (1991) Helical microtubules of graphitic carbon. Nature 354:56-58

Jakobtorweihen S, Verbeek M, Lowe C, Keil F, Smit B (2005) Understanding the loading dependence of self-diffusion in carbon nanotubes. Phys Rev Lett 95:044501

Jalili S, Majidi R (2007a) The effect of atomic hydrogen adsorption on single-walled carbon nanotubes properties. J Iran Chem Soc 4:431-437

Jalili S, Majidi R (2007b) Study of Xe and Kr adsorption on open single-walled carbon nanotubes using molecular dynamics simulations. Phys E 39:166-170

Jorio A, Dresselhaus G, Dresselhaus (2007) Carbon nanotubes: advanced topics in the synthesis, structure, properties and applications, vol 111. Springer Science \& Business Media, Berlin

Joseph S, Mashl RJ, Jakobsson E, Aluru N (2003) Electrolytic transport in modified carbon nanotubes. Nano Lett 3:1399-1403

Kahng Y, Hallock R, Dujardin E (2011) Gas adsorption on singlewall carbon nanotube bundles and charcoal samples. Phys Rev B 83:115434

Kang JW, Song KO, Hwang HJ, Jiang Q (2006) Nanotube oscillator based on a short single-walled carbon nanotube bundle. Nanotechnology 17:2250

Knippenberg MT, Stuart SJ, Cheng H (2008) Molecular dynamics simulations on hydrogen adsorption in finite single walled carbon nanotube bundles. J Mol Model 14:343-351

Kuila T, Bose S, Mishra AK, Khanra P, Kim NH, Lee JH (2012) Chemical functionalization of graphene and its applications progress in materials. Science 57:1061-1105

Lau K-t, Lu M, Lam C-k, Cheung H-y, Sheng F-L, Li H-L (2005) Thermal and mechanical properties of single-walled carbon nanotube bundle-reinforced epoxy nanocomposites: the role of solvent for nanotube dispersion. Compos Sci Technol 65:719-725 
Liu H, Cooper VR, Dai S, D-e Jiang (2012) Windowed carbon nanotubes for efficient $\mathrm{CO}_{2}$ removal from natural gas. J Phys Chem Lett 3:3343-3347

Liu L, Nicholson D, Bhatia SK (2015) Adsorption of $\mathrm{CH}_{4}$ and $\mathrm{CH}_{4} /$ $\mathrm{CO}_{2}$ mixtures in carbon nanotubes and disordered carbons: a molecular simulation study. Chem Eng Sci 121:268-278

Majidi R (2014) Molecular dynamics simulation of noble gases adsorption on carbon nanotube bundles. Fullerenes Nanotubes Carbon Nanostruct 22:520-527

Majidi R, Tabrizi KG (2010) Study of neon adsorption on carbon nanocones using molecular dynamics simulation. Phys B 405:2144-2148

Majumder M, Chopra N, Andrews R, Hinds BJ (2005) Nanoscale hydrodynamics: enhanced flow in carbon nanotubes. Nature 438:44

Majumder M, Keis K, Zhan X, Meadows C, Cole J, Hinds BJ (2008) Enhanced electrostatic modulation of ionic diffusion through carbon nanotube membranes by diazonium grafting chemistry. J Membr Sci 316:89-96

Miller SA, Young VY, Martin CR (2001) Electroosmotic flow in template-prepared carbon nanotube membranes. J Am Chem Soc 123:12335-12342

Mohammad AW, Teow YH, Ang WL, Chung YT, Oatley-Radcliffe DL, Hilal N (2015) Nanofiltration membranes review: recent advances and future prospects. Desalination 356:226-254

Novoselov KS, Falko VI, Colombo L, Gellert PR, Schwab MG, Kim K (2012) A roadmap for graphene. Nature 490:192-200

O'connell MJ (2006) Carbon nanotubes: properties and applications. CRC Press, Boca Raton

Odom TW, Huang J-L, Kim P, Lieber CM (1998) Atomic structure and electronic properties of single-walled carbon nanotubes. Nature 391:62-64

Peng F, Pan F, Sun H, Lu L, Jiang Z (2007) Novel nanocomposite pervaporation membranes composed of poly (vinyl alcohol) and chitosan-wrapped carbon nanotube. J Membr Sci 300:13-19

Porada S, Zhao R, Van Der Wal A, Presser V, Biesheuvel P (2013) Review on the science and technology of water desalination by capacitive deionization. Prog Mater Sci 58:1388-1442

Qin X, Yuan Q, Zhao Y, Xie S, Liu Z (2011) Measurement of the rate of water translocation through carbon nanotubes. Nano Lett 11:2173-2177

Reich S, Thomsen C, Ordejon P (2002) Electronic band structure of isolated and bundled carbon nanotubes. Phys Rev B 65:155411

Ren L, Wang S (2010) Tailoring optical and electrical properties of carbon nanotube networks for photovoltaic applications. Carbon 48:4397-4402

Shannon MA, Bohn PW, Elimelech M, Georgiadis JG, Marinas BJ, Mayes AM (2008) Science and technology for water purification in the coming decades. Nature 452:301-310
Srivastava A, Srivastava O, Talapatra S, Vajtai R, Ajayan P (2004) Carbon nanotube filters. Nat Mater 3:610-614

Stan G, Bojan MJ, Curtarolo S, Gatica SM, Cole MW (2000a) Uptake of gases in bundles of carbon nanotubes. Phys Rev B 62:2173

Stan G, Hartman JM, Crespi VH, Gatica SM, Cole MW (2000b) Helium mixtures in nanotube bundles. Phys Rev B 61:7288

Surapathi A, H-y Chen, Marand E, Karl Johnson J, Sedlakova Z (2013) Gas sorption properties of zwitterion-functionalized carbon nanotubes. J Membr Sci 429:88-94

Thomas JA, McGaughey AJ (2008) Reassessing fast water transport through carbon nanotubes. Nano Lett 8:2788-2793

Tian X, Wang Z, Yang Z, Xiu P, Zhou B (2013) Adsorptive separation of ethylene/ethane mixtures using carbon nanotubes: a molecular dynamics study. J Phys D Appl Phys 46:395302

Tour JM, Kittrell C, Colvin VL (2010) Green carbon as a bridge to renewable energy. Nat Mater 9:871-874

Vela S, Huarte-Larrañaga F (2011) A molecular dynamics simulation of methane adsorption in single walled carbon nanotube bundles. Carbon 49:4544-4553

Wang EN, Karnik R (2012) Water desalination: graphene cleans up water. Nat Nanotechnol 7:552-554

Wang W, Peng X, Cao D (2011) Capture of trace sulfur gases from binary mixtures by single-walled carbon nanotube arrays: a molecular simulation study. Environ Sci Technol 45:4832-4838

Weber S, Talapatra S, Journet C, Zambano A, Migone A (2000) Determination of the binding energy of methane on singlewalled carbon nanotube bundles. Phys. Rev. B 61:13150

Wei W, Qu X (2012) Extraordinary physical properties of functionalized graphene. Small 8:2138-2151

Whitby M, Quirke N (2007) Fluid flow in carbon nanotubes and nanopipes. Nat Nano 2:87-94

Whitby M, Cagnon L, Thanou M, Quirke N (2008) Enhanced fluid flow through nanoscale carbon pipes. Nano Lett 8:2632-2637

Yavari F, Koratkar N (2012) Graphene-based chemical sensors. J Phys Chem Lett 3:1746-1753

Yoo D-H, Rue G-H, Hwang Y-H, Kim H-K (2002a) Study of nitrogen adsorbed on single-walled carbon nanotube bundles. J Phys Chem C 106:3371-3374

Yoo D-H, Rue G-H, Seo J-Y, Hwang Y-H, Chan M, Kim H-K (2002b) Study of argon adsorbed on open-ended carbon nanotube bundles. J Phys Chem C 106:9000-9003

Yoo YS, Shin J, Seo I, Kim J, Lee JH, Lee SH (2015) Bending behavior of a carbon nanotube with internal fluid flow. Fullerenes Nanotubes Carbon Nanostruct 23:431-436

Yu M-F, Files BS, Arepalli S, Ruoff RS (2000) Tensile loading of ropes of single wall carbon nanotubes and their mechanical properties. Phys Rev Lett 84:5552 
Yu M, Funke HH, Falconer JL, Noble RD (2010) Gated ion transport through dense carbon nanotube membranes. J Am Chem Soc 132:8285-8290

Yzeiri I, Patra N, Král P (2014) Porous carbon nanotubes: molecular absorption, transport, and separation. J Chem Phys 140:104704
Zolfaghari A, Hashemi F, Pourhossein P, Jooya H (2007) Molecular dynamics simulations on the effect of temperature and loading in $\mathrm{H}_{2}$ exohedral adsorption on $(3,3)$ and $(9,9)$ SWCNTs. Int $\mathbf{J}$ Hydrogen Energy 32:4889-4893 